\title{
Cardiac arrhythmias in non-cardiac critically ill patients
}

\author{
Sherif H.Zaky ${ }^{(1)}$, Khalied A. Elkhashab ${ }^{(2)}$, Osama M. Momtaz ${ }^{(2)}$, Ghada M. Naguib ${ }^{(2)}$ \\ (1) Department of Critical Care Medicine,Faculty of Medicine,Cairo University,Egypt. \\ (2) Department of Critical Care Medicine,Faculty of Medicine,Fayoum University,Egypt.
}

Corresponding author:Ghada Mohamed Naguib,

E-mail:GMN00@fayoum.edu.eg

Mobile:01093632105

\section{ABSTRACT}

\begin{abstract}
Aim of the work: To evaluate the incidence and types of cardiac arrhythmias in non-cardiac critically ill patients admitted to the general ICU and the relation of arrhythmias to different comorbidities and patient outcomes.
\end{abstract}

Patients and methods: prospective study on 72 non-cardiac critically ill patients admitted to the general intensive care unit of Critical Care Department of Fayoum University Hospital from May 2015 to November 2016 presenting with different causes of admission.

Results: 136 episodes of arrhythmias occurred in all of the studied patients, the most

\section{KEY WORDS:}

Arrhythmia, Intensive care unit, NonCardiac, Mortality.

\section{Introduction}

Arrhythmias are a common problem in the critically ill and they can have significant effects on patient outcome. They often require frequent was sinus tachycardia as it occurred in 72 non-cardiac patients in the form (30.15 $\%$ of attacks were sinus tachycardia) followed by $22.97 \%$ for atrial extrasystoles, $14.71 \%$ for ventricular extrasystole, $9.56 \%$ for SVT, $9.56 \%$ for AF, $8.09 \%$ for MAT. Sinus tachycardia was the most common to occur and mortality incidence was $34.7 \%$ among the study group.

Conclusion: Supraventricular arrhythmias were more common to occur in non-cardiac critically ill patients than ventricular arrhythmias. Patient with AF developed in the setting of critical illness carries higher propensity to mortality

immediate and swift action and it is, therefore, essential that clinicians have a structured approach to the recognition and management 
of arrhythmias. The normal heart rate (HR) is between 60 and 100 beats per minute (bpm). The heart beat provides the mechanical force for the pumping the oxygenated blood to, and deoxygenated blood away from, the peripheral tissues. This depends critically on the orderly activation and recovery of electrical excitation through the myocardium. Disruptions of this can lead to arrhythmias.

Understanding of the mechanisms underlying their generation and maintenance requires knowledge of the ionic contributions to the cellular action potential. Arrhythmia only rarely appears to be a diagnosis for primary admission to the ICU (1). Rather, arrhythmias occur during the ICU stay. It is

\section{PATIENTS AND METHODS:}

Seventy two critically ill non cardiac patients who were admitted to the general intensive care units of Critical Care Department of ElFayoum University Hospital due to different acute presentation were studied during the period from May 2015 to November 2016. An informed written consent was taken from all patients or their close relatives.

\section{Patient selection:}

In all admitted patients, 72 patients who got

(a) sustained tachyarrhythmias (more than 30 seconds);

(b) all achyarrhythmias requiring therapy; evident that the occurrence of arrhythmia will depend on an underlying disease different etiologies, such as chronic obstructive pulmonary disease, acute respiratory distress syndrome, pulmonary embolism, and valvular heart disease, may lead to arrhythmia the ICU environment,(2) with interventions such as mechanical ventilation, vasopressors, and inotropes or invasive procedures, may be the cause of arrhythmia. Although sympathetic tone definitely is a trigger for arrhythmias and sudden cardiac death (3), the influence of analgesia and sedation. Common operative procedures in critical illness might predispose to arrhythmia. One process common to critical illness and multiorgan failure of different origin is systemic inflammation.

(c) bradycardias of fewer than 40 beats/min or requiring intervention

(d) extrasystoles (atrial or ventricular), during the ICU admission were selected and thoroughly studied. Age was selected from 1880 years, both sex were included. Patients who met one or more of these criteria were excluded:

evident rheumatic heart disease, cardiomyopathy, myocardial, endocardial or pericardial diseases secondary to other systemic disease, suspected coronary artery disease, regional hypokinesia, left ventricular systolic dysfunction by echocardiograpy. 
All included patients were subjected to full clinical evaluation, Vital signs, Acute

Physiology And Chronic Health Evaluation

(APACHE II scoring),full laboratory

investigations,Continuous ECG

monitoring,Echo Doppler imaging: using the ACUSON CV70 Echo-Doppler machines equipped with a $2.5 / 3.25-\mathrm{MHZ}$ annular array transducer. Conventional M-mode and twodimensional parasternal and apical windows were performed. Measurement of the left ventricular echocardiography from a left ejection fraction was performed by Simpson's method (8) and also by M-mode by measuring LV systolic and diastolic dimensions obtained through long-axis parasternal approach in Mmode projection.

\section{Statistical Analysis}

Data were collected and coded to facilitate data manipulation and double entered into

\section{RESULTS:}

This study was conducted on 72 non-cardiac patients (out of 850 patients totally admitted in ICU) with percentage of $8.5 \%$ with 136 arrythmia episodes. 41 males with mean age $(48.9 \pm 16.5)$ years. Mean Acute Physiology And Chronic Health Evaluation (APACHE II scoring) (52.8 \pm 9.7$)$ Admission diagnoses were 20 patients $(27.7 \%)$ septic shock, 18 patients (25\%) stroke, 18 patients (25\%) respiratory failure (I,II), 10 patients (13.8\%) DKA , 4 patients $(5.5 \%)$ status epilepticus and 2 patients $(2.7 \%)$ hepatic cell failure.
Microsoft Access and data analysis was performed using SPSS software version 18 in windows 7.Simple descriptive analysis in the form of numbers and percentages for qualitative data, and arithmetic means as central tendency measurement, standard deviations as measure of dispersion for quantitative parametric data, and inferential

\section{statistic test:}

For quantitative parametric data :Independed student t-Test used to compare measures of two independent groups of quantitative data.For quantitative non parametric data :Mann-whitney test in comparing two independent groups.

For qualitative data:Chi square test to compare two of more than two qualitative groups. The level $\mathrm{P} \leq 0.05$ was considered the cut-off value for significance.

Group means of potassium ,sodium and calcium were within normal range.however,hypokalemia of $3.5 \mathrm{meq} / \mathrm{l}$ or lower was noted on 40 instances, hyponateriemia of $134 \mathrm{meq} / \mathrm{l}$ or less was noted on 16 instances ,hypocalcemia of $8.5 \mathrm{meq} / \mathrm{L}$ was noted on 40 instances .

Blood gases analysis noted acidosis in $(51.3 \%)$ of episodes, alkalosis in $9.7 \%$ of episodes, hypercapenia in $(20 \%),(75 \%)$ of patients were hypoxic. In this study there were 136 episodes of arrhythmias, in the form of 
$30.15 \%$ sinus tachycardia, $22.97 \%$ atrial extrasystole, $14.71 \%$ ventricular extrasystole , 9.56 SVT, 9.56\% for AF, 8.09\% for MAT and least frequency was to sinus bradycardia, atrial flutter, and Ventricular Bigimini arrhythmia see figure(1)

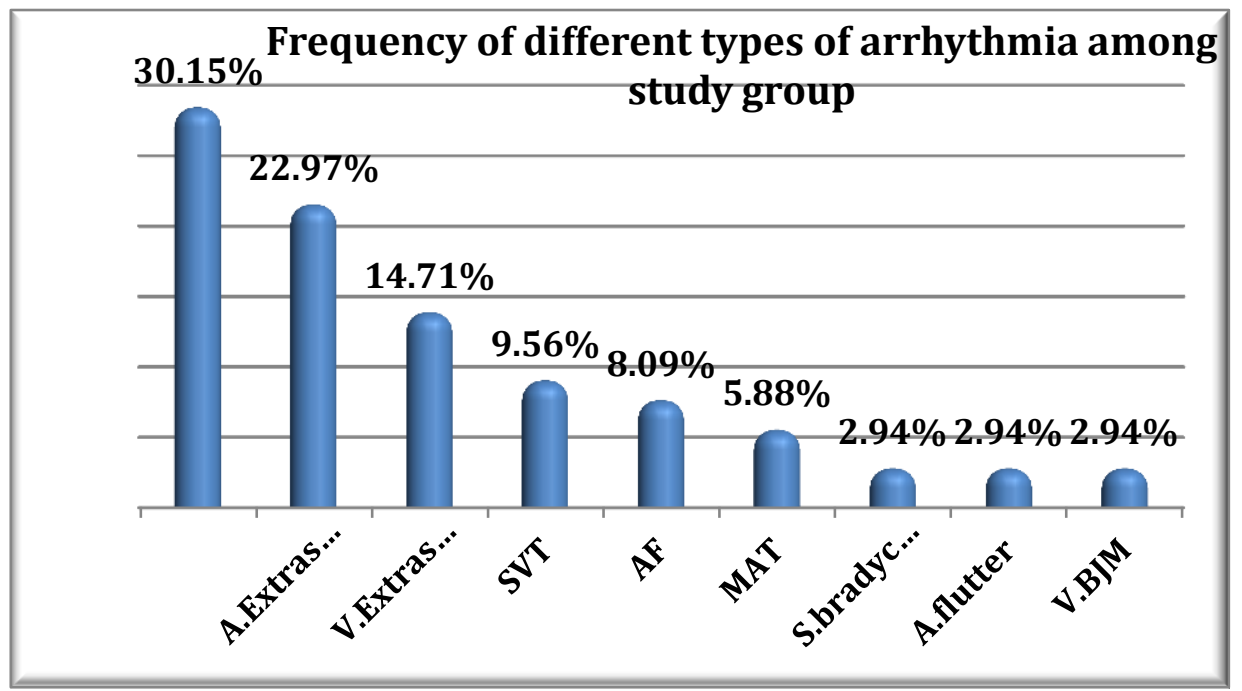

Figure (1): Frequency of different types of arrhythmia among study group

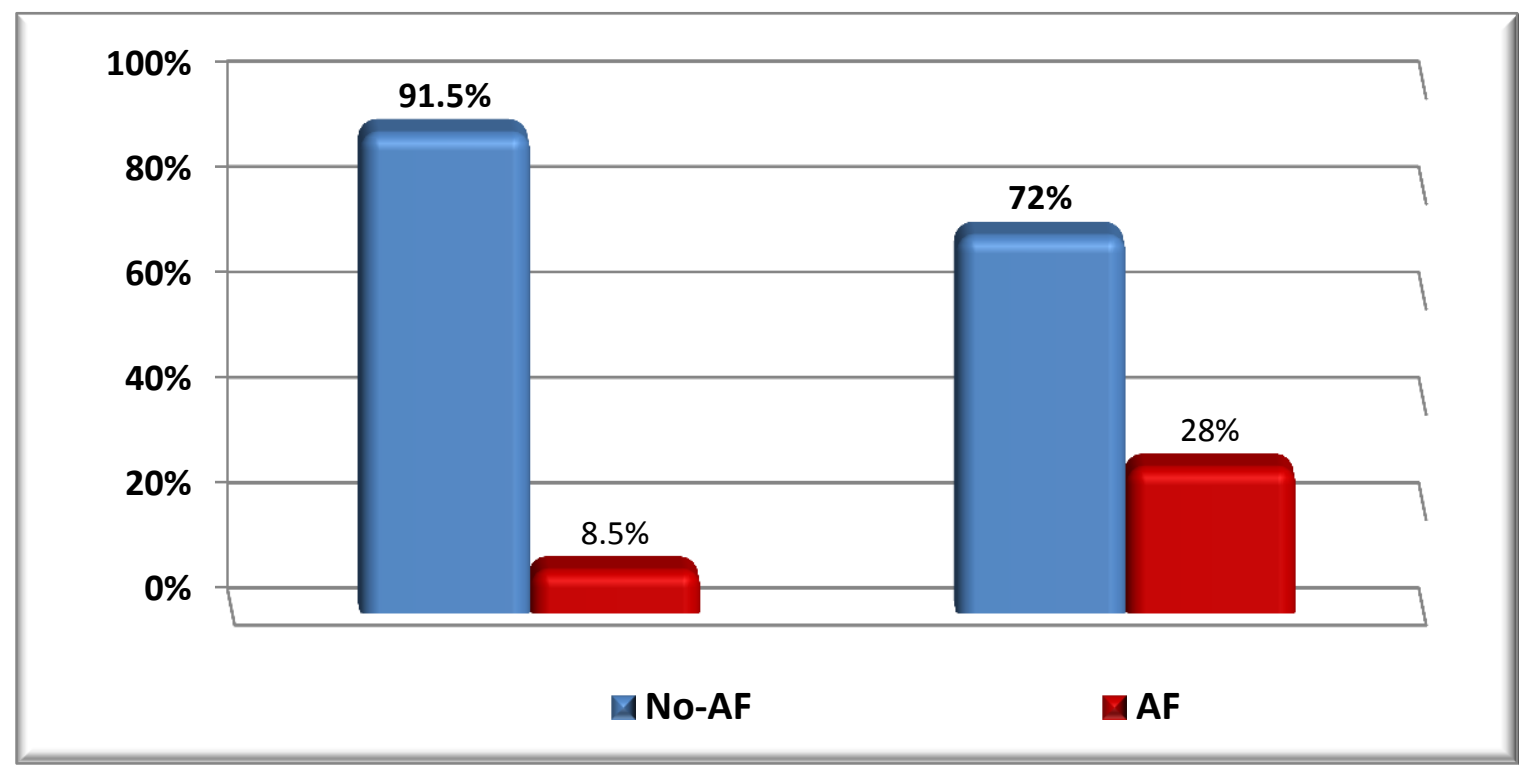

\section{Survived}

Not-Survived

Figure (2): Outcome of patients with AF

there is statistically significant difference with p-value $<0.05$ in APACHE II score with high mean among AF, patients, and low mean among ventricular extrasystole and Ventricular bigimini patients. Also there is statistical significant high mortality rate among patients with AF, and atrial flutter arrhythmic patients see figure (2). 


\section{DISCUSSION}

This study was done on 72 non-cardiac patients admitted in ICU, (56.9\%) were males, with mean age of $(48.9 \pm 16.5)$ years, ranged between 16 and 69 years old .One hundred thirty six (136) episodes of arrhythmias were recorded, sinus tachycardia was the most common(30.15\%), , $\mathrm{AF}(9.5 \%)$.The mortality incidence was $34.7 \%$ among the whole study group with statistical significant high mortality rate among patients with $\mathrm{AF}(\mathrm{P}=$ 0.04). APACHE II score was high among the whole study group (mean=41.6 \pm 14.1 ) higher among AF patients $(\mathrm{P}=0.001)$.

In comparsion to the study done in Bursa, Turkey by G Tasdemir et al in 2015, in Uludag University Medical ICU to evaluate incidence and risk factors of arrhythmias occurring in patients in the ICU excluding cardiac valvular disease, cardiac surgery in the recent 6-month period, history of myocardial infarction. Two hundred and fourteen patients were included in the study twenty-six percent $(n=56)$ of patients had arrhythmias. Incidence was higher in females $(\mathrm{P}=0.045)$. Average age of arrhythmic patients was 69. APACHE II scores were higher in arrhythmic patients $(\mathrm{P}=0.001)$. Atrial fibrillation was the most frequent type of arrhythmia. The mortality rate for all patients was $48.1 \%$. There was no statistically significant relationship between arrhythmia and mortality $(\mathrm{P}>0.05)$. arrhythmia, found in $32 \%$ of patients.

In the present study, there was 136 episodes of arrhythmias occurred in all of the studied patients, the most frequent was sinus tachycardia as it occurred in the form $(30.15 \%$ of attacks were sinus tachycardia) followed by $22.97 \%$ for atrial extrasystoles, $14.71 \%$ for ventricular extrasystole, $9.56 \%$ for SVT, 9.56\% for AF, $8.09 \%$ for MAT and least frequency was to sinus bradycardia, atrial flutter, and Ventricular Bigimini .Only twenty four $(17.6 \%)$ episodes of wide complex arrhythmias out of 136 episodes were recorded ,none of them was ventricular fibrillation .

In contrast to the study conducted in 2001 by Gottfried Heinz et al, in mixed ICU on 133 patients with sustained arrhythmias(extrasystoles were excluded)that showed 310 arrhythmia episodes,278 tachyarrhythmias(108(34\%) narrow QRS complex,168(54\%) wide QRS complex),48.6 ventricular,29.8 atrial fibrillation,3.6\% atrial flutter,7.5\% supraventricular tachycardia.

The difference in this study to our study may be referred to the fact that they included mainly cardiac patients, post cardiothoracic surgery patients and post myocardial infarction. In addition extrasystoles were excluded in their study. 
In current study, it is noticed that $22.2 \%$ (16) of patients were hyponateriemic $(\mathrm{Na}<134$ $\mathrm{mEq} / \mathrm{L}), 55 \%$ (40) were hypokalemic $(\mathrm{K}<3.5$ $\mathrm{mEq} / \mathrm{L}) 55 \%$ (40) patients with total calcium ( $<8.8 \mathrm{mg} / \mathrm{dL}), 40.2 \%$ (29) patients with ionized calcium $(<4.5 \mathrm{mg} / \mathrm{dL})$.

In contrast to the study done in 2001 by Gottfried Heinz et al in mixed ICU on 133 patients with sustained arrhythmias that showed 310 arrhythmia episodes, hypokalemia was found in 32(24\%) episode, and hypomagnesaemia in 36 (27\%) episode.

It was noticed that current study group patients had higher APACHE II scoring compared to other studies conducted on incidence of arrhythmia in critically ill patients. Patients with new onset AF presented with (mean $\pm \mathrm{SD})$ APACHI II score (52.8 \pm 9.7$)$, hypotension $(72.7 \%)$, (mean \pm SD) mean arterial blood pressure (MAP) (57.3 \pm 16.6$)$, (63.6\%) on vasopressors. our critically ill noncardiac patients with new onset AF had higher APACHE II scoring, lower MAP, higher mortality incidence (63.6\%) among the study group and in comparison to most of the studies.

In 2012, Salmaan Kanji et al., did their study on 3081 medical and noncardiac surgical

\section{CONCLUSION}

Cardiac arrhythmias occur frequently in critical non-cardiac patients admitted in the general medical ICU. critically ill patients in three mixed ICU at Ottawa Hospital Research Institute. A total of 3081 patients were admitted during the 1-year study period in which 348 consecutive patients (10.5\%) had documented AF. Hemodynamic instability developed in $37 \%$ and $10 \%$ of patients with new-onset AF and patients with preexisting AF, respectively. Atrial fibrillation was of new onset in 139 patients (4.5\%) and preexisting in 186 patients $(6.0 \%)$.

Patients with new-onset AF presented with (mean \pm SD) $22.6 \pm 9.0$ APACHE II score with ; Hypotension (46\%), on Vasopressors ( $40 \%)$, Septic shock (27\%), Heart failure with pulmonary edema (12\%), Myocardial infarction $(6 \%)$. The use of vasopressors was limited only to nineteen patients out of seventy two critically ill cases.

Obviously, the incidence of arrhythmia was rather correlated to the severity of the critical illness rather than the effect of medications used to compact this illness and managing its threatening nature (vasopressors or electrolyte disturbance imbalance had minimal correlation to arrhythmia incidence compared to APACHE II score through direct or indirect effect on inflammatory mediators affecting arrhythmia incidence.

The most frequent arrhythmias are sinus tachycardia, atrial extrasystoles, ventricular extrasystole, SVT, MAT AF, and atrial 
flutter.Patients with AF and atrial flutter carry

higher mortality.Identifying the most common association with serious arrhythmias may have the potential in tailoring meticulous management of the causative factors in one

\section{REFERANCES}

[1] Kanno S, Saffitz JE. The role of myocardial gap junctions in electrical conduction and arrhythmogenesis. Cardiovasc Pathol 2001;10:169-77

[2] Chung MK, Martin DO, Sprecher D,Wazni O, KanderianA, Carnes CA Bauer JA, Tchou PJ, Niebauer MJ, Natale A, et al. C-reactive protein elevation in patients with atrial arrhythmias: inflammatory mechanisms and side and the arrhythmia itself on the other side to achieve better patient outcome.We recommend studying this issue on a wider cohort of patients. persistence of atrial fibrillation. Circulation 2001;104:2886-2891.

[3] Seguin P, Laviolle B, Maurice A,

Lecqulerc C, Malledant Y. Atrial fibrillation in trauma patients requiring intensive care.

Intensive Care Med 2006;32:398-404.

[4] Heinz G. Circadian variation in ventricular tachycardia and atrial fibrillation in a medicalcardiological ICU. Intensive Care Med 2003;23. 